\title{
A REVIEW ON ANALYTICAL METHOD DEVELOPMENT AND VALIDATION
}

\author{
SHIVANI SHARMA ${ }^{*}$, SWAPNIL GOYAL ${ }^{2}$, KALINDI CHAUHAN ${ }^{3}$ \\ 1Department of Pharmaceutical Quality Assurance, ${ }^{2}$ Deparment of Pharmacology, ${ }^{3}$ Department of Pharmaceutics, Mandsaur University, \\ Mandsaur (M. P.), India \\ Email: shivanisharma327@gmail.com \\ Received: 03 Jul 2018, Revised and Accepted: 04 Sep 2018
}

\begin{abstract}
The top objective of any pharmaceutical industry is to produce products of necessary characteristic and quality reliably, in a cost-effective manner. Development of a method is essential for discovery, development, and evaluation of medicines in the pharmaceutical formulation. The main aim of this review article was to check the development and validation of the procedure employed for the medication from the starting of the formulation to the complete commercial batch of product. At the point when an analytical technique is applied to produce outcomes for the quality of medicine associated samples, it is necessary that the outcomes are reliable. In the pharma industry, validation policy is documented for how to perform validation, types of validation and validation policy are complied with the necessities of good manufacturing practice (GMP) regulations. Validation is very important for the effective running of the pharmaceutical firms. At every stage from raw material to the finished, stability, everywhere validation was performed. The method was developed properly, and validation parameters are explained in terms of accuracy, specificity, precision, limit of detection (LOD), limit of quantitation (LOQ), ruggedness, robustness, and system suitability testing with the example of certain drugs. All validation parameters are used in the routine and stability analysis.
\end{abstract}

Keywords: Validation, Method development, Limit of quantitation, Limit of detection, Linearity, Robustness, Ruggedness

(C) 2018 The Authors. Published by Innovare Academic Sciences Pvt Ltd. This is an open-access article under the CC BY license (http://creativecommons.org/licenses/by/4.0/) DOI: http://dx.doi.org/10.22159/ijap.2018v10i6.28279

\section{INTRODUCTION}

Analysis is vital in any product or service, and it is also important in drug because it involves life [1]. Analytical chemistry is the analysis of separation, quantification and chemical additives identification of herbal and synthetic materials constituted with one or more compounds or factors. Analytical chemistry is separated into two predominant classes, a qualitative evaluation that is to say the identification with regard to the chemical additives exists in the sample, whereas quantitative evaluation estimates the amount of positive detail or compound within the substance, i.e. the sample [2]. The quantity of medication brought into the marketplace is growing each year. These medicines could also be either new entities or partial structural alteration of the present one. Medicines ought to be accessible in such a type that quality as well as bioavailability, adequate plasma concentration, desired period, the onset of action, correct dose, safety, effectiveness and stability on storage of product, will be assured throughout the storage of the products [3]. The advancement of a medication is a long procedure including drug invention, a research lab trial, preclinical testing, clinical testing, and regulatory registration. To additionally improve the adequacy and protection of the medication after acceptance, numerous administrative organizations, for example, the united states food and drug administration (USFDA) likewise require that the drug product is evaluated for its identification, potency, characteristics, quality, stability, and purity before it can be discharged for utilizing. Thus, pharmaceutical validation and process controls are vital in disregarding the issues that might be encountered [4]. Frequently, there is a time lag from the date of introduction of a medication into the market to the date of its consideration in pharmacopeias. This occurs because of the possible vulnerabilities within the continuous and more extensive use of those drugs, reports of ongoing toxicity (bringing about their withdrawal from the market), development of affected person resistance and improvement of higher medicine with the aim of competition. Under these situations, requirements and analytical techniques for those medicines that may not be approachable within the pharmacopoeias. It becomes necessary, so as to develop newer analytical strategies for such medications [5]. Analytical approach improvement and validation perform important functions in the discovery, improvement, and manufacturing of medications. The main aim of an analytical measure is to get consistent, realistic, and correct information. Validated analytical strategies play a significant role in achieving this goal. Outcomes from methodology validation may be used to choose the standard, reliability, and consistency of analytical results, that is associated as an integral part of any sensible analytical practice. Validation of analytical strategies is also needed by most rules and quality standards that impact laboratories [6].

\section{Analytical method development}

When there are no definitive techniques are present, new methodologies are being progressed for evaluation of the novel product. To investigate the presence of either pharmacopoeial or non-pharmacopoeial product novel techniques are developed to reduce the value besides time for higher precision and strength. These methodologies are optimized and valid through preliminary runs. Alternate ways are planned and place into practice to exchange the present procedure within the comparative laboratory information with all accessible merits and demerits.

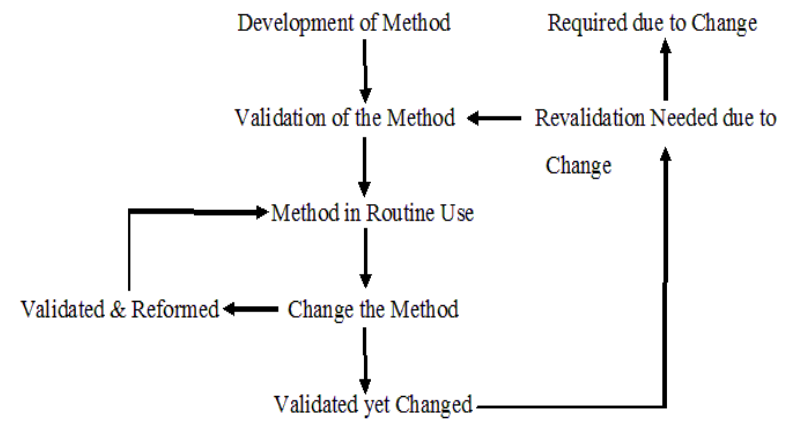

Fig. 1: Life cycle of the analytical method [7]

\section{Necessity of method development}

Drug evaluation exhibits the identity characterization and resolution of the drugs in combination like dosage forms and organic fluids. At some point of producing technique and development of drug the principal purpose of analytical strategies is to generate data regarding efficiency (which might be directly connected with the need of a identified dose), impurity (related to safety of the 
medication), bioavailability (consists of key drug traits like crystal kind, uniformity of drug and release of drug), stability(that shows the degradation product), and effect of manufacturing parameters to verify that the production of drug product is steady.

Analyst before the development of new technologies, do not forget below mention criteria:

- Is this technique possesses the needful sensitivity?

- Is this method sufficiently selective for direct use without interference by means of the opposite element within the sample?

- Is the accuracy and precision doable with this technique?

- Are the reagents and equipment required on this method available or obtained at a reasonable price?

- Is the time requires to perform this technique applicable [3]?

\section{Steps for developing a method}

Various steps are involved in the development of an analytical method are as follows:

\section{$>$ Characterization of analyte and standard}

- All the known necessary data concerning the analyte and its structure that is to mention the physical and chemical properties such as solubility, optical isomerism, etc., are collected.

- The standard analyte is equal to $100 \%$ purity is acquired. Necessary arrangement is to be created for the proper storage (refrigerator, desiccators, and freezer).

- In the sample matrix, when multiple parts are to be measured the amount of elements is observed duly presenting the information and the accessibility of standard are calculated.

- Techniques like spectroscopy (UV-Visible, FTIR, atomic absorption spectroscopy, etc.), high-performance liquid chromategraphy and gas chromatography so on and, are however about once coordinated with the stability of samples [2].

$>$ Requirement of the technique: Requirement of analytical methodology is essential to build up the analytical fig. of advantage like linearity, selectivity, specificity, range, accuracy, precision, LOD, LOQ etc. shall be outlined [2].

$>$ Literature survey and prior methods: All the data of literature related to the drug are reviewed for its physical and chemical properties, manufacturing, solubility and applicable analytical ways with reference to relevant books, journals, united states pharmacopeia/national formulary(USP/NF), association of official agricultural chemists (AOAC) and american society for testing and materials (ASTM) publications and it is extremely convenient to look Chemical Abstracts Service automatic computerized literature [2].

\section{$>$ Selecting the method}

- Utilizing the data obtained from the literature, the methodology is evolving since the method is being modified wherever needed. Sometimes, it is important to acquire additional instrumentation to create, alter or replicate and validate existing procedures for analytes and tests.

- If there are not any past appropriate ways available to investigate the analyte to be examined [2].

$>$ Proper instrumentation and initial studies: Installation qualification (IQ), operation qualification (OQ), and performance qualification $(\mathrm{PQ})$ of instrument pertinent to research standard methodology is examined by an appropriate set up of instruments [2].

> Optimization: While performing optimization, once a parameter is modified at a time, and a group of conditions are differentiated, before utilizing trial and error approach. This work is needed for accomplished basing on a scientific organized method plan duly all necessary points and documented with relation to dead ends [2].
Proper documentation of analytical fig. of merits: The true determined analytical fig. of benefit consisting of LOD, LOQ, cost, linearity and evaluation time and planning of samples, etc. are also recorded [2].

> Evaluation of produced technique with actual specimen: The specimen solution needs to prompt specific, complete recognition of the peak interest of the medication other than all different matrix parts [2].

$>$ Estimation of percent recovery of real samples and demonstration of quantitative sample analysis: Percentage recovery of spiked, actual standard medication into a sample grid which includes no analyte is evaluated. Optimization to reproducibility of recuperation from test to test must have appeared. It is not always essential to get $100 \%$ restoration so far as the outcomes are reproducible to perceive with a high level of assurance [2].

\section{Validation}

Validation is an idea that has developed in the U. S. in 1978. The idea of validation has extended during that time to grasp an extensive variety of activities from analytical approaches utilized for the quality control of medication to computerized systems for clinical trials, marking or process control, validation is established on, however not endorsed by regulatory specifications and is best seen as a critical and necessary part of current good manufacturing practice (cGMP).

The phrase validation basically implies for evaluation of validity or activity of demonstrating viability. Validation is a workforce effort where it entails humans from various departments of the plant. Validation is needed for any new or amended technique to confirm that it is capable of giving consistent and reliable results, once utilized by different operators using similar instrumentation within the same or completely different laboratories [8]. Validation is an essential component of quality assurance; it includes the efficient investigation of systems, facilities, and procedures aimed toward deciding if they execute their planned capacities sufficiently and reliably as determined.

Validation should in this way be considered in the accompanying circumstances:

- Completely new procedure.

- Latest equipment.

- Procedure and equipment which have been adjusted to suit altered needs and,

- Procedure where the finished result test is a poor and undependable marker of product quality [4].

\section{Important stages in validation}

The action identifying with validation studies can be categorized mainly into three stages:

\section{Stage 1}

This includes pre-validation qualification stage which covers all exercises identifying with product studies and improvement, formulation pilot batch testing, scale-up research, exchange of innovation to business scale groups, setting up stability conditions, and managing of in-process, finished pharmaceutical formulations, qualification of equipment, master documents, and process limit [4].

\section{Stage 2}

This involves process validation phase. It is intended to check that every installed limit of the vital process parameter is substantial and that satisfactory products can be created even below the worst situations [4].

\section{Stage 3}

It is also called as the validation maintenance stage, it requires constant review of all procedure related archives, including validation of the review reports, to guarantee that there have been 
no modifications, departure, failures, and alteration to the production procedure and that all standard operating procedures (SOPs), involving change control procedures, had been observed. At this phase, the approval team involving people representing all essential departments also guarantees that there have been no modifications/deviations that ought to have brought about requalification and revalidation [4].

Types of validation

Validation is classified into following types:

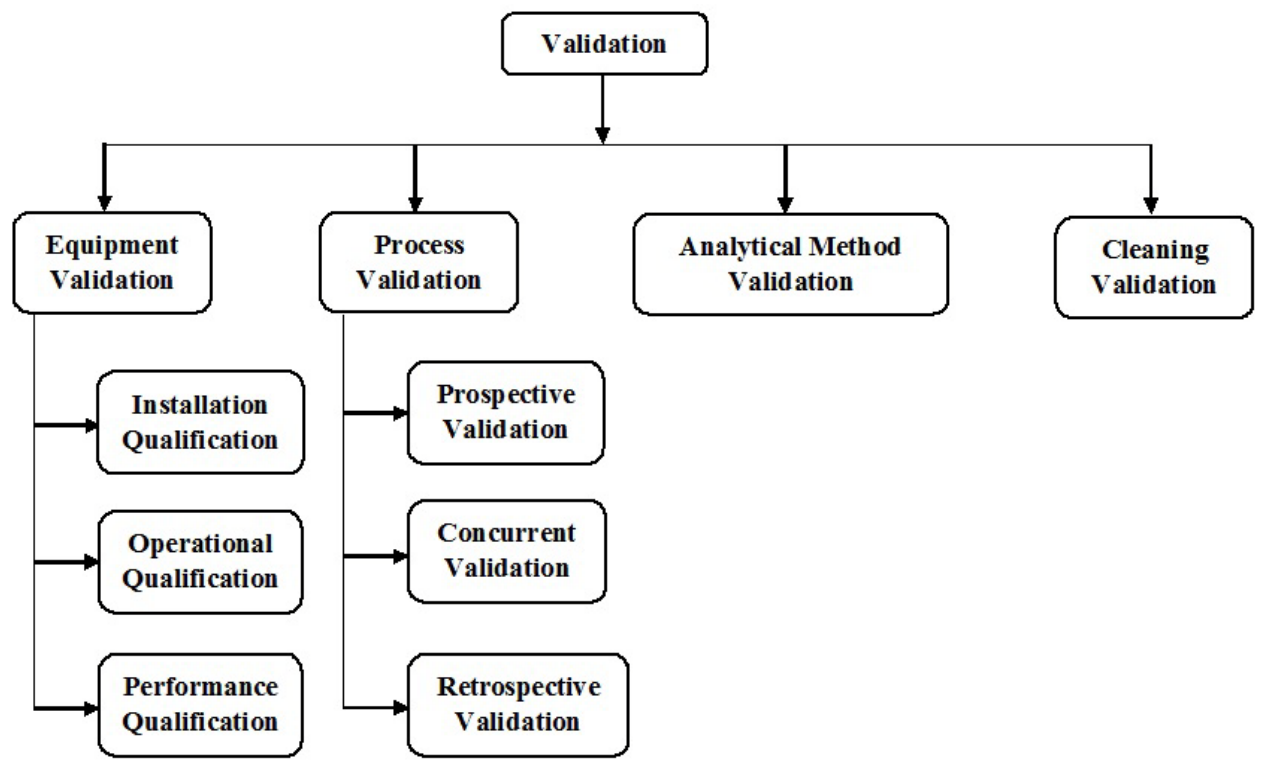

Fig. 2: Validation types [9]

\section{Equipment validation}

The key concept of validation is to give a high degree of reported confirmation that the equipment and the procedure conform to the written guidelines. The degree (or intensity) is dictated by the complexity of the device or system. The validation should give the essential data and test methods required to give that the device and technique meet determined prerequisites. Equipment Validation includes the following:

Installation qualification (IQ): IQ guarantees all crucial processing, packaging system, and ancillary items are in compliance with the installation. It checks that the equipment has been established or installed as per the manufacturer's suggestion in a systematic way and positioned in surrounding appropriate for its meant purpose [10].

Installation qualification points include

- Equipment layout character that is the material of construction cleanability and many others.

- Installation situations like wiring, functionality, utility and so forth.

- Calibration, preventative protection, cleansing plans.

- Safety characteristics.

- Supplier documentation, prints, illustrations, and hand operated.

- Software documentation.

- Enlist the spare components.

- Environment-related conditions like clean room necessities, humidity, and temperature [11].

$>$ Operational qualification (OQ): OQ performed to give a high level of degree of affirmation that the equipment works as proposed [10].

OQ concerns consist of:

- Process control limits like temperature, time, stress, line velocity, set up conditions, and so on.
- Software parameters.

- Crude material details.

- Process operating methods.

- Material managing necessities.

- Process change control.

- Training.

- Short-term balance and capability of the technique.

- The use of statistically valid procedures inclusive of screening examinations to optimize the technique can be utilized throughout this stage [11].

Performance qualification (PQ): PQ checks that the device is repeatable and it is uniformly producing a quality item [10]

$P Q$ concern consists of:

- True product, procedure parameters, and process set up in $\mathrm{OQ}$

- Adequacy of the product.

- Guarantee of technique ability as built up in $\mathrm{OQ}$

- Process repeatability, prolonged process stability [11].

\section{Process validation}

The process validation is a component of the coherent prerequisites of a quality management system [11]. Process Validation is the most essential and perceived parameters of current good manufacturing practices. The objective of a quality system is to produce items that are matched with their proposed use uniformly. Process approval is a key component in guaranteeing that these standards and objective are met.

$>$ Process validation is reported evidence which gives a high level of affirmation that a particular procedure will produce a product meeting its determined prerequisites. It mainly involves the following [8] 
Prospective validation: It is described as the well-known reported program that a device does what it indicated to do based on pre-planned protocols. This validation is normally performed previously for distribution both of a newer item or item made under a revised production process [12].

In this validation, the protocol is accomplished before the procedure is placed into industrial use [8].

Prospective validation ought to incorporate, however, not be limited to the subsequent:

- Short depiction of the procedure.

- Summary of the important processing steps to be evaluated.

- Equipment/facilities list is to be utilized (involving calculation, observing/recording equipment) collectively with its calibration status.

- $\quad$ Finished dosage forms for discharge.

- List of analytical techniques, as suitable.

- Proposed in-process controls with specification criteria.

- Additional testing to be completed, with specification limits and analytical approval, as suitable.

- Sampling design.

- Techniques for recording and assessing outcomes.

- Functions and obligations.

- $\quad$ Proposed timetable [13].

$>$ Concurrent validation: It is same as prospective validation with the exception of the working firm, will offer the product at the time of qualification runs, to the society at its market cost, and furthermore like retrospective validation. This type of validation includes in-process observing of vital processing steps and product checking out. This helps to produce and reported proof to demonstrate that the manufacturing technique is in a condition of control [12].

This approval includes in-process observing of essential processing steps and product testing. This creates and recorded proof to demonstrate that the production procedure is in a condition of the control.

- In remarkable conditions, it might be acceptable not to finish the validation program before routine manufacturing begins.

- The choice to complete simultaneous approval must be supported, archived and accepted by authorized personnel.

- Documentation prerequisites for simultaneous validation are similar as designated for prospective validation [13].

$>$ Retrospective validation: It is characterized by the established reported confirmation that a system does what it implies to do on the audit and investigation of historical data. This is accomplished by the survey of the ancient manufacturing testing information to demonstrate that the procedure has always remained in control. This kind of approval of a procedure for an item already in distribution. Retrospective validation is adequate for wellestablished procedures and will be wrong where there have been current modifications within the composition of the product, working methods or device [12].

Few basic components of retrospective validation are:

- Batches are produced for a definite duration (last 10 successive batches). The number of lots discharged every year.

- Batch size/strength/producer/year/period.

- Master manufacturing/packaging files.

- Current particulars for active ingredients/finished materials.
- List of process deviations, corrective actions, and modification to production archives.

- Data for stability study for a few batches [13].

$>$ Revalidation: Revalidation gives the proof that modifications in the procedure, as well as the procedure condition that are presented don't unfavorably influence process attributes and product quality. Organizations, facilities, equipment and methods which include cleaning, ought to be periodically assessed to affirm that they stay valid. Where no remarkable modifications have been made to the approved status, a review with proof that facilities, organizations, equipment and procedures address the recommended necessities satisfies the need for revalidation [13].

Revalidation becomes vital in specific circumstances. Few of the modifications that require validation are mentioned below:

- Modifications in crude materials.

- Modifications in the equipment.

- Modifications in the source of active crude material producer.

- Alteration of packing material.

- Modification of the procedure.

- Modifications inside the plant/facility.

- A selection is no longer to carry out revalidation studies have to be completely justified and reported [13].

\section{Analytical method validation}

Validation of an analytical approach is established through laboratory research, that the execution attributes of the procedure meet the requirements for the proposed scientific application. Validation is required for any new or altered procedure to verify that it is fit for giving predictable and dependable outcomes, once used by various administrators by usage of comparable instrumentation inside the similar or absolutely distinct laboratories [14].

Method validation is a reported program that offers with that the processing system will give a high level of affirmation to meet its predicated acceptance basis [9].

It consists of mainly five different steps which are as follows:

$>$ Qualification of the system: System qualifications permit to check that the instrument is appropriate for the planned investigation, the materials are appropriate to be used in analytical judgments, the analysts have the correct instruction, capabilities, and foregoing documentation such as analytical inclusive of analytical approaches, proper authorized protocol with pre-set up standards have been reviewed. On the off chance that the general qualifications of a device are overlooked, and trouble arises, the source of the issue will be hard to recognize [15].

$>$ Sampling: Sampling assists in the choice of a representative part of the fabric which is along these lines subjected to evaluation. The selection of a suitable sampling technique is of significant importance since it gives assurances that the sample chose is really illustrative of the material as a whole for the purpose of important statistical inferences. Inside the statistical literature, there is a considerable collection of work on sampling techniques, anyway the relative expenses and time engaged with every technique ought to be assessed ahead of time [15].

$>$ Preparation of sample: Preparation of the sample is a key component to effective method validation. It has been mentioned that sample planning represents 60 to $80 \%$ of the work action and working expenses in an investigative lab. The literature on the preparation of the sample is enough and properly documented. In any case, the investigator ought to recall that the choice of a particular preparation technique relies upon concentrations of analytes, sample matrix, size of the sample and the instrumental method [15]. 
Analysis of sample: The evaluation is associated with the instrument utilized to extract qualitative or quantitative data from the samples with an adequate vulnerability level. The investigation could be predictable, in a great sense, as the device has 3 interconnected fundamental components, namely input, converter, and output. The input and output are assigned by the letters $\mathrm{x}$ and $\mathrm{y}$, and they represent the concentration and response individually. The selection of a specific analysis depends on many considerations, for example, the chemical properties of the analytical species, the concentration of the analytes in the sample, sample matrix, speed, cost, and so forth [15].

$>$ Assessment of data: The essential reason behind information assessment is to outline and pick up knowledge into a specific informational index by utilizing numerical and statistical techniques. Data assessment permits extracting valuable data and reaching inferences about the inputs and outputs, and in particular about the validation procedure in general [15]

\section{Cleaning validation}

Cleaning validation is a reported proof with a high level of confirmation that can uniformly clean a system or equipment to already determined and specification criteria. Cleaning approval is a reported procedure that demonstrates the efficacy and consistency in cleaning pharmaceutical production equipment. The goal of cleaning approval is to check the viability of the cleaning system for the expulsion of product deposits, degradants, additives, excipients, or cleaning agents and in a the control of potential microbial contamination.

It is vital to validate cleaning techniques for the following motives:

- Pharmaceutical products and active pharmaceutical ingredient (API) can be contaminated by other products and microbes.

- It is an administrative prerequisite in pharmaceutical product manufacture the worry is the same-guarantee that the equipment is properly clean and safety and quality is kept up.

- It is likewise guaranteed from an inside control and consistency perspective the quality of manufacture.

- To protect product integrity.

- To reuse the equipment $[16,17]$.

\section{Necessity for cleaning validation}

To check the viability of cleaning techniques and to make sure that no risks are related to cross-contamination of API or detergents $[16,17]$.

\section{Cleaning validation protocol}

- The goal of the validation procedure.

- Obligations regarding performing and endorsing the validation study.

- Equipment details.

- The interval between the end of production and the start of the cleaning techniques.

- Cleaning methods to be utilized for every product, each manufacturing device or each piece of equipment.

- The quantity of the cleaning cycle to be performed continuously.

- Routine checking equipment.

- Sampling techniques, including the basis for why a specific sampling technique is utilized.

- Clearly defined sampling areas.

- Information on recovery studies, where suitable.

- Analytical techniques including LOD and LOQ.

- The acceptance criteria, along with including the method of reasoning for setting specified limits [18].

\section{Importance of validation}

- Assured high quality.

- Time boundation.

- Optimization of the method.

- Minimum batch product failure, enhanced efficiency, manufacturing, and productivity.

- Quality cost decreased.

- Rejection decreased.

- Yield increases.

- Fewer complaints about process related issues.

- Fast and realistic start-up of new equipment's.

- Increased worker consciousness of the process [9].

\section{Validation parameters}

The main aim of method validation is to produce proof that the method will what it is supposed to do, accurately, reliable and consistent [9]. The validation parameters as per ICH guidelines are described below:

$>$ Accuracy: Accuracy is expressed as the nearness of agreement between the values found and values that are already available. It can also be defined as the closeness between the true value and the observed value. It is sometimes called as trueness, and it could be determined by using at least 9 determinations over a minimum of 3 concentration over the specified range [19].

Accuracy of prochlorperazine maleate (PRO) and betahistine hydrochloride (BET) was studied by the standard addition method at three different levels (50\%, 100\%, and 120\%). A known amount of drug was added to the pre-analyzed sample and percentage recovery calculated. When this method was used for accuracy, the recovery was found to be $99.38 \%$ for betahistine hydrochloride and 99.11\% for prochlorperazine maleate [20].

For the concurrent determination of nitazoxanide and ofloxacin accuracy was studied by the standard addition method at five different levels $(50 \%, 75 \%, 100 \%, 125 \%$, and $150 \%)$. The results indicate that the recoveries were observed to be in the range of $80 \%$ to $120 \%$, therefore, the method is accurate [21].

Accuracy of paracetamol was studied by preparing standard solution of different concentrations $(10,35,55 \mu \mathrm{g} / \mathrm{ml})$ and injected to check the $\%$ recovery. The percent recovery of the drug was found in the range of 98.8 to $102.0 \%$ respectively for all concentrations [22]

$>$ Precision: The exactness of an analytical procedure expresses the nearness of agreement (degree of scatter) between a group of measurements obtained from different sampling of a uniform sample underneath the prescribed conditions [23] Precision may be taken into consideration at 3 levels:

- Repeatability: It expresses the exactness below a similar operating condition over a brief interval of time and also referred as intra-assay precision. A minimum of six replicates test preparation of a similar or consistent sample ready at the $100 \%$ check [24].

- Intermediate precision: It expresses the exactness under inside research laboratories, in distinct days, through distinct analyst, on distinct instruments/equipment. Two different analysts each preparing six sample solutions, as per specified method [25].

- Reproducibility: It refers to the precision between different analytical labs. Every research facility set up an aggregate of six sample solutions, according to the analytical technique [24].

The precision of prochlorperazine maleate (PRO) and betahistine hydrochloride (BET) method was determined by interday and intraday variation (\% RSD). Intra-day precision was performed by analyzing standard drug solutions within the calibration range, three times on the same day. Inter-day 
precision was performed by analysing drug solutions within the calibration range on three different days over a period of seven days. The low \% RSD values of interday $(1.02$ to $1.48 \%$ for BET at $252.9 \mathrm{~nm}$ and 0.67 to $0.82 \%$ for PRO at $260.15 \mathrm{~nm}$ ) and intraday ( 0.77 to $1.09 \%$ for BET at $252.9 \mathrm{~nm}$ and 0.27 to $0.61 \%$ for PRO at $260.15 \mathrm{~nm}$ variation for BET and PRO, revealed that the method is precise [20].

For simultaneous estimation of nitazoxanide and ofloxacin precision performed by injecting six replicates of a sample prepared from commercial tablets and assay was calculated to determine the repeatability of retention time and a peak area of standard and samples. The percentage relative standard deviation (\% RSD) values for the area of nitazoxanide and ofloxacin were 0.44 and $0.2 \%$ and RSD values for a retention time of nitazoxanide and ofloxacin were $0.44 \%$ for both the drugs [21].

The precision of paracetamol was checked by injecting a solution of $80 \mu \mathrm{g} / \mathrm{ml}$ for six times in same days, different days, and in a different time interval on the same day. The \% RSD was found to be less than $3 \%$, which showed good precision [22].

$>$ Specificity: For every stage of development, the analytical technique should demonstrate specificity. The technique was should have the power to unequivocally assess the analyte of interest whereas within the presence of all expected parts, which can encompass degradants, excipients/sample matrix, and sample blank peaks [26].

Specificity was performed to determine the retention time of each drug in a mixture and in the sample. The retention time of standard drugs individually was determined, and it was found to be $3.750 \mathrm{~min}$ and $1.533 \mathrm{~min}$ for nitazoxanide and ofloxacin and retention time of both drugs in the standard mix was found to be 3.760 min for nitazoxanide and $1.542 \mathrm{~min}$ for ofloxacin respectively [21].

$>$ Limit of detection (LOD): Lowest quantity of an analyte which may be detected by the chromatographical separation however it is not necessary that this quantity will quantify as a precise value. A blank resolution is injected and peak to peak quantitative noise relation we have to calculate from blank chromatograms. Then, calculate the concentration at the signal to quantitative noise relation is concerning 3:1.

LOD can be expressed as

$\mathrm{LOD}=3.3 \mathrm{SD} / \mathrm{S}$

Where, $\mathrm{SD}=$ Standard deviation of response,

S = Slope of calibration curve [27].

The LOD value of betahistine hydrochloride (BET) and prochlorperazine maleate (PRO) is $0.29 \mu \mathrm{g} / \mathrm{ml}$ and $0.34 \mu \mathrm{g} / \mathrm{ml}$ respectively [20].

LOD of the paracetamol was studied by the signal to noise ratio, and the result was found to be $120 \mu \mathrm{g} / \mathrm{ml}$ [22].

$>$ Limit of Quantitation (LOQ): It is characterized by the least quantity of an analyte that can be quantified with exactness and precision.

LOQ can be communicated as

$\mathrm{LOQ}=10 \mathrm{SD} / \mathrm{S}$

Where SD = Standard deviation of response,

$\mathrm{S}=$ Slope of calibration curve [28].

The LOQ value of betahistine hydrochloride (BET) and prochlorperazine maleate (PRO) is $0.957 \mu \mathrm{g} / \mathrm{ml}$ and $1.12 \mu \mathrm{g} / \mathrm{ml}$ respectively [20].

LOQ of the paracetamol was studied by the signal to noise ratio, and the result was found to be $360 \mu \mathrm{g} / \mathrm{ml}$ [22].
Some usual techniques, methods for the assessment of LOD and LOQ are as follows:

- Visual inspection,

- Signal to noise ratio,

- Standard deviation of the blank, and

- Regression line at low concentrations [29].

> Linearity: Linearity may be characterized as the capacity of an analytical technique to produce outcomes which are directly related to the concentration of an analyte in the [30].

Standard solution of betahistine hydrochloride (BET) and prochlorperazine maleate (PRO) was taken in a $10 \mathrm{ml}$ volumetric flask and diluted with $0.1 \mathrm{~N}$ HCL to get the final concentration in the range of 4 to $24 \mu \mathrm{g} / \mathrm{ml}$ for BET and 3 to $18 \mu \mathrm{g} / \mathrm{ml}$ for PRO. Prepared six times in this calibration range and absorbance determined at the respective wavelength for each drug alone. The results show good linearity between absorbance and concentration in the prescribed concentration range for both the drugs [20].

For simultaneous estimation of nitazoxanide and ofloxacin linearity perform as five different concentrations of standard mixtures prepared, 50\%, 75\%, 100\%, 125\%, 150\% were injected, and chromatogram was recorded. The correlation coefficient was calculated and was observed to be greater than 0.99 for both the drugs which are within the limit [21].

The linearity of paracetamol was performed by preparing different concentrations $(6.25,12.5,25,50$, and $100 \mu \mathrm{g} / \mathrm{ml})$ from a stock solution of $10 \mathrm{mg} / \mathrm{ml}$. The solution of $20 \mu \mathrm{l}$ was injected into column three times. Linearity of paracetamol was found in the concentration range of $6.25-100 \mu \mathrm{g} / \mathrm{ml}$ with a correlation coefficient of 0.999 [22].

$>$ Range: It can be characterized as the interval amongst upper and lower quantities of analyte in the sample. Minimum of the specified range to be $80 \%$ to $120 \%$ of the test sample for the assay test [31].

$>$ Ruggedness: Ruggedness is the degree or measure of reproducibility under different situations such as in different laboratories, different analyst, different machines, environmental conditions, operators etc. [32]

In the simultaneous estimation of nitazoxanide and ofloxacin ruggedness was performed by different analyst and in different laboratories in different days to checks for any variation in the chromatography. The \% RSD for area and retention time was calculated for determination [21].

$>$ Robustness: It is characterized by the level of ability of an analytical technique, to stay similar by minute purposely change in the technique parameter. The different technique parameters which can be modified in high-performance liquid chromatography are $\mathrm{pH}$, drift rate, the temperature of the column and mobile phase composition [33].

In the simultaneous estimation of nitazoxanide and ofloxacin, the robustness of the proposed method verified by to perform analysis under variable flow rates. The flow rate as per the developed method is $1.5 \mathrm{ml} / \mathrm{min}$. Slight change in flow rate is $1.3 \mathrm{ml} / \mathrm{min}$ and $1.7 \mathrm{ml} / \mathrm{min}$ and chromatogram recorded. Due to a slight change in the flow rate of method shows good results and remain unaffected by that minute change. So, we can say that the method is robust [21].

For robustness of paracetamol, small modification in the flow rate, $\%$ of acetonitrile and $\mathrm{pH}$ of the mobile phase, the method remains unaffected and \% RSD value obtained are approximately near [22].

$>$ System suitability parameters: System suitability test is used to check the sensitivity, resolution, and reproducibility of the chromatographic system are well for the analysis to be done. The factors mainly used in system suitability are tailing factor, a number of the theoretical plate, retention time, resolution, etc. [34]. 
Table 1: Acceptance criteria of system suitability parameters

\begin{tabular}{lll}
\hline S. No. & Parameter name & Acceptance criteria \\
\hline 1. & Tailing Factor & $<2$ \\
2. & Number of theoretical plate & $>2000$ \\
3. & Resolution & $>1.5$ \\
4. & RSD & $<2$ \\
\hline
\end{tabular}

- Tailing factor (T): It is defined as the distance between the front edge of the peak to the back edge of the peak divided by twice of the front edge of the peak.

$$
\mathrm{T}=(\mathrm{X}+\mathrm{Y}) / 2 \mathrm{X}
$$

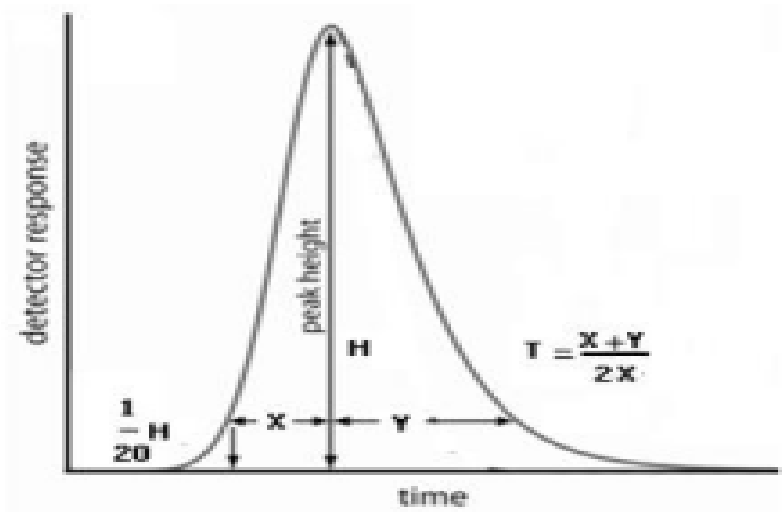

Fig. 3: Tailing factor [35]

Where $\mathrm{X}=$ Front edge of the peak and,

$\mathrm{Y}=$ Back edge of the peak [35].

- Capacity factor (K)): It can be expressed as how many times the analyte is retained with respect town retained compound. It is denoted by the symbol $\mathrm{k}$.

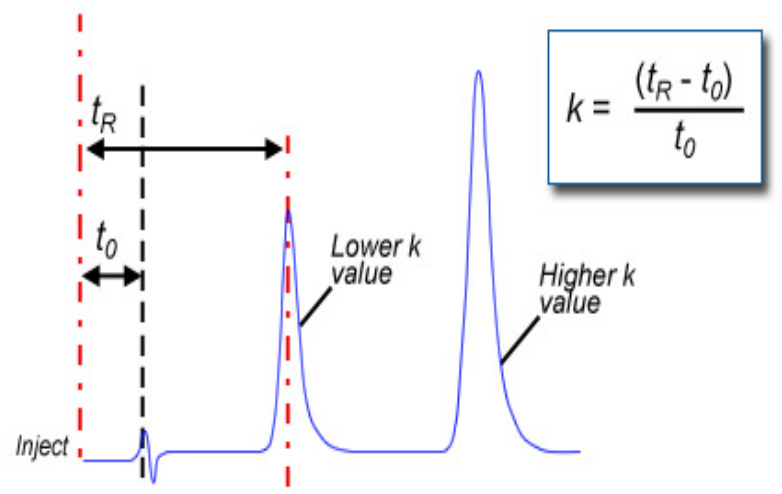

Fig. 4: Capacity factor [36]

It can be calculated as:

$\mathrm{K}=\left(\mathrm{t}_{\mathrm{R}}-\mathrm{t}_{0}\right) / \mathrm{t}_{0}$

Where $t_{R}$ is the retention time of the peak and $t_{0}$ is the column dead time [27]

It is mainly utilized to examine the efficiency of the column. It can be expressed as:

$\mathrm{N}=16\left(\mathrm{t}_{\mathrm{R}} / \mathrm{W}\right)^{2}$
Where $t_{R}$ is the retention time, and $\mathrm{W}$ is the width at the base of peak [35].

- Retention time $\left(t_{R)}\right.$ : It is the time of elution of the peak after injection of the compound. The retention time of compounds will vary depending upon:

Temperature of the column,

Composition of solvents,

Nature of stationary used and,

Pressure used [37].

- Resolution (R): It is the measure of separation power of the complete chromatographic system. Resolution can be defined as the ratio of the distance between two peak maxima to the mean value of peak width from its baseline.

$\mathrm{R}=2\left[\left(\mathrm{t}_{\mathrm{R}}\right)_{\mathrm{A}-}\left(\mathrm{t}_{\mathrm{R}}\right)_{B}\right] / \mathrm{W}_{\mathrm{A}}+\mathrm{W}_{\mathrm{B}}$

Where, $t_{R 1}$ and $t_{R 2}$ are retention time of second and first compounds, respectively [35].

Peak Resolution

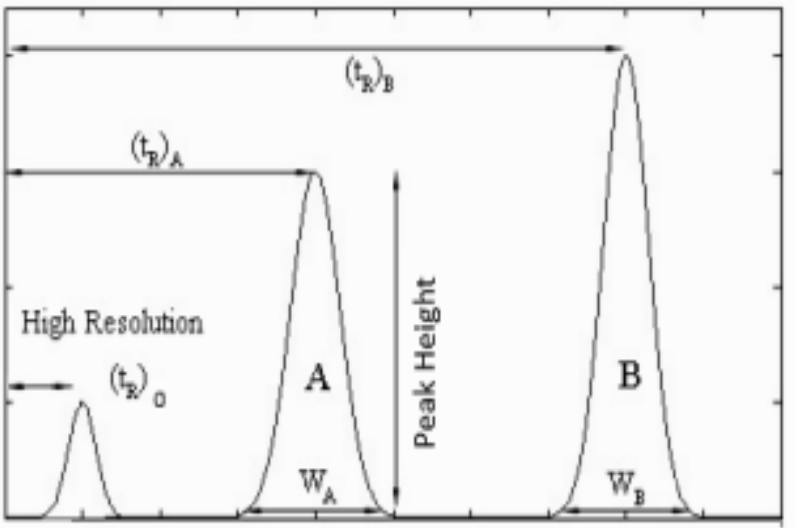

Fig. 5: Resolution [35]

In the simultaneous estimation of nitazoxanide and ofloxacin system suitability parameters was checked by injecting five injections of standard mixed solutions and two injections of the sample. The \% RSD of both the drugs is less than $0.2 \%$ [21].

\section{CONCLUSION}

This article gives an idea that what is validation, its types, why it is necessary, how to develop a method and how to carry out the validation procedure to demonstrate that the technique is able for its proposed reason. All validation parameters such as linearity, LOQ, LOD, Range, specificity, robustness, ruggedness and system suitability are defined well with examples of certain drugs. Validation is a necessary technique in the pharma department, and it is used to assure that the quality is worked into the procedures supporting the development of drug and production. 


\section{AUTHORS CONTRIBUTIONS}

All the authors have contributed equally

\section{CONFLICT OF INTERESTS}

There is no conflict of interest from all the authors

\section{REFERENCES}

1. Hema, Swati Reddy G. A review on new analytical method development and validation by RP-HPLC. Int Res J Pharm Biosci 2017;4:41-50.

2. Ravisankar P, Navya CN, Pravallika D, Sri DN. A review on stepby-step analytical method validation. IOSR J Pharm 2015;5:7-19.

3. Patel A, Dwivedi N, Kaurav N, Bashani S, Patel S, Sharma HS, et al. Chemical analysis of pharmaceuticals: a review. J Med Pharm Innov 2016;3:4-7.

4. Jatto E, Okhamafe AO. An overview of pharmaceutical validation and process controls in drug development. Trop Pharm Res 2002;1:115-22.

5. Pathuri R, Muthukumaran M, Krishnamoorthy B, Nishat A. A review on analytical method development and validation of the pharmaceutical technology. Curr Pharm Res 2013;3:855-70.

6. Patil R, Deshmukh T, Patil V, Khandelwal K. Review on analytical method development and validation. Res Rev J Pharm Anal 2014;3:1-10.

7. Chauhan A, Mittu B, Chauhan P. Analytical method development and validation: a concise review. J Anal Bioanal Tech 2015;6:1.

8. Mahar P, Verma A. Pharmaceutical process validation: an overview. Int J Pharm Res Biosci 2014;3:243-62.

9. Lavanya G, Sunil M, Eswarudu MM, Eswaraiah MC, Harisudha K, Spandana BN, et al. Analytical method validation: an updated review. Int J Pharm Sci Res 2013;4:1280.

10. Verma P, Madhav NS, KR Gupta V. A review article on pharmaceutical validation and process controls. Pharma Innovation 2012;1:51-60.

11. Md Alamshoaib. Pharmaceutical process validation: an overview. J Adv Pharm Edu Res 2012;2:185-200.

12. Ahir KB, Singh KD, Yadav SP, Patel HS, Poyahari CB. Overview of validation and basic concepts of process validation. Sch Acad J Pharm 2014;3:178-90.

13. Nandhakumar L, Dharmamoorthy G, Rameshkumar S, Chandrasekaran S. An overview of pharmaceutical validation: quality assurance viewpoint. Int $\mathrm{J}$ Res Pharm Chem 2011;1:1003-14.

14. Bhardwaj SK, Dwivedi K, Agarwal DD. A review: HPLC method development and validation. Int J Anal Bioanal Chem 2015;5:76-1.

15. Araujo P. Key aspects of analytical method validation and linearity evaluation. J Chromatogr B 2009;877:2224-34.

16. Goyal D, Maurya S, Verma C. Cleaning validation in the pharmaceutical industry-an overview. Pharma Tutor 2016;4:14-20.
17. Lodhi B, Padamwar P, Patel A. Cleaning validation for the pharmaceuticals, biopharmaceuticals, cosmetic and neutraceuticals industries. J Innov Pharm Biol Sci 2014;1:27-38.

18. Murthy DN, Chitra K. A review article on cleaning validation. Int J Pharm Sci Res 2013;4:3317.

19. Srivastava RK, Kumar SS. An updated review: analytical method validation. Eur J Pharm Med Res 2017;4:774-84.

20. Patel Paresh U. Development and validation of simultaneous equations method for estimation of betahistine dihydrochloride and prochlorperazine maleate in tablet dosage form. Inventi Rapid: Pharm Analysis and Quality Assurance; 2013.

21. Nirupa G, Tripathi UM. RP-HPLC analytical method development and validation for simultaneous estimation of two drugs nitazoxanide, ofloxacin and its pharmaceutical dosage forms. Int J ChemTech Res 2012;5:775-83.

22. Devi TP, Setti A, Srikanth S, Nallapeta S, Pawar SC, Rao JV, et al. Method development and validation of paracetamol drug by RP-HPLC. J Med Allied Sci 2013;3:8.

23. Nayudu ST, Suresh PV. Bio-analytical method validation-a review. Int J Pharm Chem Res 2017;3:283-93.

24. Daksh S, Goyal A, Pandiya CK. Validation of analytical methodsstrategy and significance. Int J Res Dev Pharm Life Sci 2015;4:1489-97.

25. Tijare LK, Rangari NT, Mahajan UN. A review on bioanalytical method development and validation. Asian J Pharm Clin Res 2016;9:6-10.

26. Tiwari G, Tiwari R. Bioanalytical method validation: an updated review. Pharm Methods 2010;1:25-8.

27. Pasbola K, Chaudhary M. Updated review on analytical method development and validation by HPLC. World J Pharm Pharm Sci 2017;6:1612-30.

28. Geetha G, Raju KN, Kumar BV, Raja MG. Analytical method validation: an updated review. Int J Pharm Biol Sci 2012;1:64-1.

29. Shrivastava A, Gupta VB. Methods for the determination of limit of detection and limit of quantitation of the analytical methods. Chron Young Sci 2011;2:21.

30. Ravichandran V, Shalini S, Sundram KM, Harish R. Validation of analytical methods-strategies and importance. Int J Pharm Pharm Sci 2010;2:18-2.

31. Shweta K, Anita S. A review on analytical method validation. Int J Pharm Res Rev 2016;5:30-6.

32. Boque R, Maroto A, Riu J, Rius FX. Validation of analytical methods. Grasas Aceites 2002;53:128-43.

33. PushpaLatha E, Sailaja B. Bioanalytical method development and validation by HPLC: a review. J Med Pharm Innov 2014;1:1-9.

34. Gupta V, Jain AD, Gill NS, Gupta K. Development and validation of HPLC method-a review. Int Res J Pharm Appl Sci 2012;2:17-5.

35. Bose A. HPLC calibration process parameters in terms of system suitability test. Austin Chromatogr 2014;1:4.

36. http://www.chromacademy.com. [Last accessed on 19 Jun 2018]

37. Tiwari P, Singh BK. HPLC: a modern approach of development and validation. World J Pharm Res 2016;5:1616-31. 